\title{
Three-Dimensional Interactive Graphical Model of the Hindlimb Muscles of the Rat
}

\author{
Guus C. Baan Huub Maas \\ Department of Human Movement Sciences, Vrije Universiteit Amsterdam, Amsterdam, The Netherlands
}

\section{Keywords}

Anatomy $\cdot$ Hindlimb $\cdot$ Muscle model $\cdot$ Rat

\begin{abstract}
Many questions in human movement sciences are addressed by exploiting the advantages of animal models. However, a 3D graphical model of the musculoskeletal system of the frequently used rat model that includes a sufficient level of detail does not exist. Therefore, the aim of the present work was to develop an freely accessible 3D graphical model of the rat hindlimb. Using the anatomical data of the Wistar rat (Mus norvegicus albinus) published by Greene [1935], a 3D representation of 34 muscles of the hindlimb was drawn. Two models were created, one using muscle-like appearances and one using different colors. Each muscle can be viewed separately or within the context of its synergistic and antagonistic muscles. This model can serve to train new students before starting their experiments but also for producing illustrations of experimental conditions or results. Further development of the model will be needed to equip it with the same advanced functionalities of some of the human anatomy atlases.

(C) 2022 The Author(s).

Published by S. Karger AG, Basel
\end{abstract}

\section{Introduction}

Anatomy is the basis for most, if not all, studies on human and animal movement. Knowledge about the location of structures within our body, their morphology, and relationship with other subsystems is essential for the design of experiments, both from a theoretical and a practical perspective. For the field of motor control, for example, one should have received training in the anatomy of the nervous system, but also of its effectors (i.e., the skeletal muscles). In biomechanics, the relationship between the muscular system and the skeleton is crucial. Only when knowing the location of the origin and insertion of muscles, their function can be understood. That is why the students that enter our lab will first receive training in the anatomy relevant for the study before starting their experiments.

Many questions in movement sciences can be addressed with measurements on human subjects, but certain questions can only be addressed when exploiting the advantages of animal models. In fact, much of our current knowledge about the muscular, skeletal, and nervous systems is based on experiments using cats, rats, and mice. For these animals, comprehensive anatomical atlases exist [such as Greene, 1935; Crouch, 1969]. An atlas together with dissections on cadavers can be used as preparation for experiments. In addition to that, $3 \mathrm{D}$ graphical models can be very helpful for training purposes. Note that graphical models differ from 3D biomechanical models of the musculoskeletal system, which include information such as the coordinates of muscle origins and insertions on the skeleton and moment arms, like those published for the rat [Young et al., 2019], mouse [Charles et al., 2016], and cat [Burkholder and Nichols, 2004].
C 2022 The Author(s).

Published by S. Karger AG, Basel

This is an Open Access article licensed under the Creative Commons Attribution-NonCommercial-4.0 International License (CC BY-NC) (http://www.karger.com/Services/OpenAccessLicense), applicable to the online version of the article only. Usage and distribution for commercial purposes requires written permission.
Correspondence to:

Huub Maas, h.maas@vu.nl 


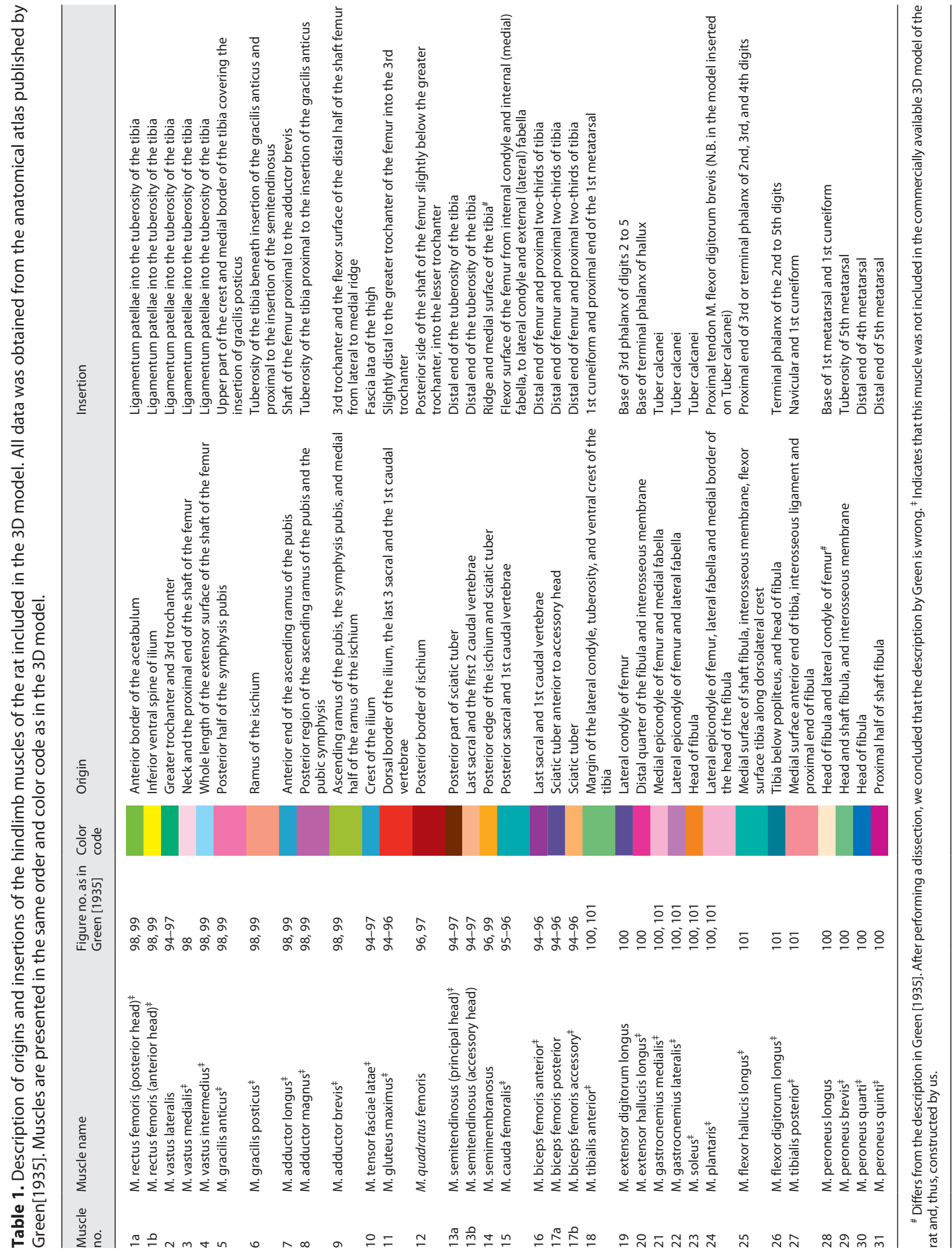




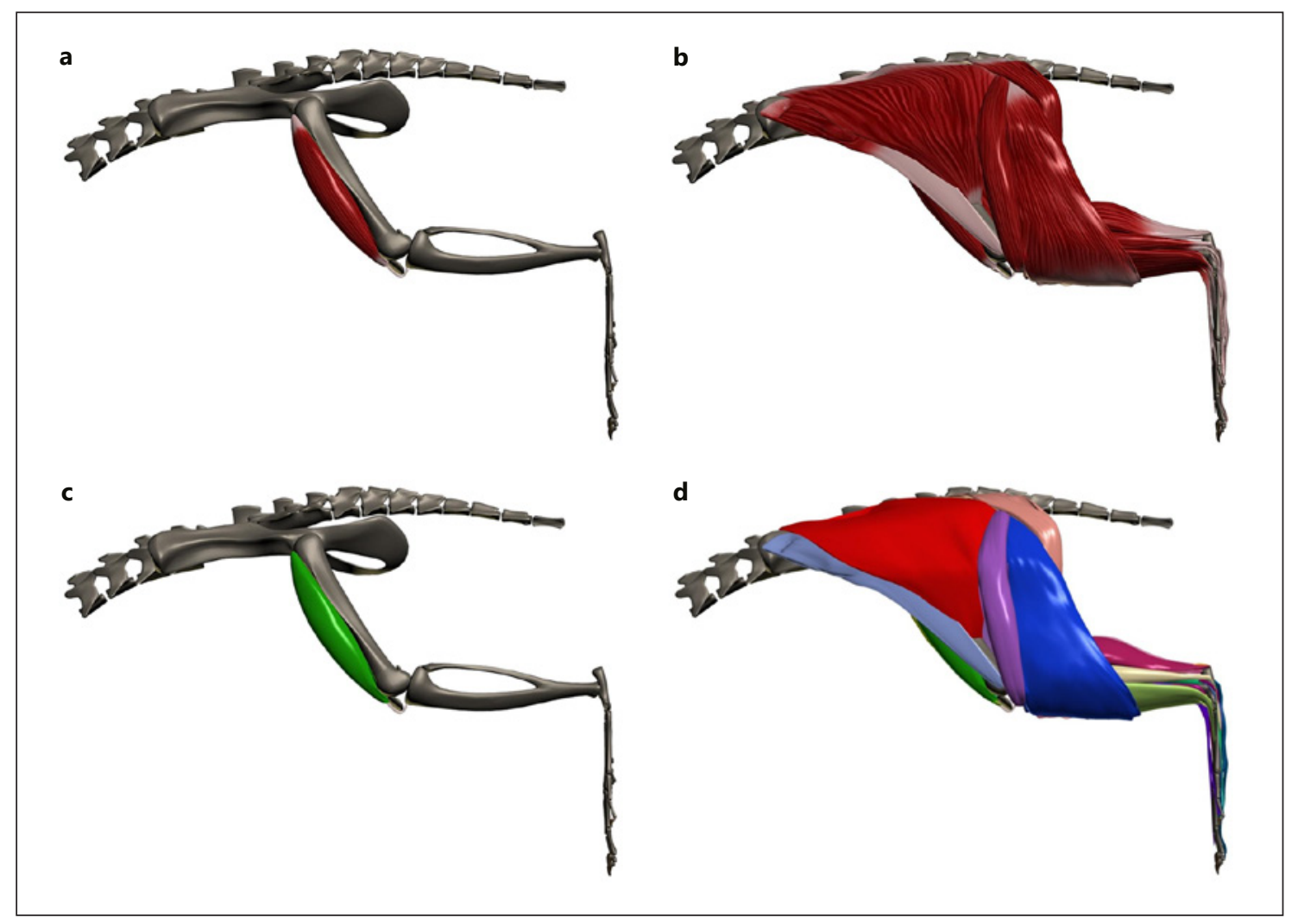

Fig. 1. Exemplar images from the model with a single muscle (M. vastus lateralis) (a, c) and all muscles (b, d) with the muscle textures (top) or the muscles in different colors (bottom).

Many of the current textbooks on human anatomy and other online platforms provide interactive $3 \mathrm{D}$ software to explore our body. We have made many 3D drawings of muscles, tendons, and peripheral nerves of the rat, often integrated in drawings of the experimental setups used for studying the mechanical and sensory action of skeletal muscles [e.g., Smilde et al., 2016; Tijs et al., 2016; Finni et al., 2018]. However, a 3D graphical model of the musculoskeletal system of frequently used animal models, the rat in particular, that includes a sufficient level of detail does not exist. Therefore, the aim of the present work was to develop a freely accessible 3D graphical model of the rat hindlimb.

\section{Model Creation}

The 3D drawings of the skeleton and muscular system were based on the commercially available $3 \mathrm{D}$ model of the rat from Turbosquid (www.turbosquid.com). For the muscles, we first obtained the location of the origin and insertion of 34 muscles of the hindlimb using the anatomical atlas of the Wistar rat (Mus norvegicus albinus) published by Greene [1935]. The muscles in the foot were not included. The information extracted is shown in Table 1 and presented graphically in online Supplement 1 (for all online suppl. material, see www.karger.com/ doi/10.1159/523708). Subsequently, the 3D representation of each muscle was remodeled to obtain an accurate representation of the muscle's origins and insertions, and to adjust their length and morphology to a different hindlimb position (i.e., ankle $90^{\circ}$, knee $120^{\circ}$, hip $120^{\circ}$ ). The majority of muscles (27 out of 34) were not included in the original model and, hence, constructed (see Table 1). For this the 3D modelling program Anim8or ${ }^{\circledR}$, which is freeware that can be downloaded from www.anim8or.com, was used. For construction of muscles that were not part of the commercially available model, we used the following sources: (1) the description of the location of origin and insertion as well as the drawings in the anatomical atlas of Greene [1935] and (2) images of the muscles taken during dissection or physiological mea- 
surements in our laboratory. When all 34 muscles were drawn, they were merged into one final model (online Suppl. 2). Besides a model using muscle textures, a second model was created in which each muscle has a unique color (online Suppl. 3).

\section{Model Functionality}

The 3D model consists of a model of each muscle separately (for example see Fig. 1a, c) and a model of all muscles combined (Fig. 1b, d). In the full hindlimb model, specific muscles can be shown by making the texture or color of individual muscles semi- or fully transparent. Each viewpoint of the model can be saved as an image for other purposes, such as scientific publications or lecture slides. The model was built to be used in Anim8or ${ }^{\circledR}$ (only compatible with Windows) but can also be used in other programs such as Blender (compatible with Mac OS). Specific guidelines for using the model can be found in online Supplement 4.

\section{Concluding Remarks}

We presented the first anatomically accurate 3D graphical model of the rat hindlimb, including all major muscles. Specific muscles can be visualized and the model can be rotated in all directions. 3D graphical models of the rat are commercially available, but none of these models include the majority of hindlimb muscles in an anatomically correct way. Although our model is a considerable improvement with respect to the current commercially available models of the rat, it has several limitations: (1) The model is not dynamic, but static in a sense that joint angles cannot be changed easily to obtain other body positions. It would be valuable to be able to explore changes in shape of different muscles while rotating a joint. (2) The muscles are presented in a very simplified view, namely with a red muscle belly and white tendinous ends connecting to the skeleton (see muscle texture model, Suppl. 2). Real muscles do not only have tendons but also aponeuroses that in many cases cover large parts of the muscle belly surface [Zuurbier et al., 1994; Haberfehlner et al., 2016; Siebert et al., 2017]. We plan to improve the accuracy of muscle morphological properties in future models. (3) Finally, our model is not as elaborate as some of the most recent 3D atlases of human anatomy, which include advanced functionalities such as virtual and augmented reality.
In our experience, obtaining a 3D picture of the muscles that are the subject of the experiment helps in mastering the surgical skills needed to prepare the muscles for physiological testing and, thus, the model can serve to train new students before starting their experiments. Like physical rat simulators [Corte et al., 2021], using our 3D model may reduce the number of animals used for scientific experiments as well as for education and training, thereby contributing to the $3 \mathrm{R}$ principle (Refine, Reduce, Replace). For this, it is crucial that the model is anatomically correct. In addition, in many cases the description of surgical and/or experimental procedures and/or results can be improved by illustrations. This model can also serve this purpose.

\section{Acknowledgements}

We thank Guido Weide, Fangxin Xiao, Guido Geusebroek, and Cintia Rivares for providing feedback on earlier versions of the model and for helping with the practical guidelines for using the model in Blender.

\section{Statement of Ethics}

For this manuscript, no experiments on live animals or tissue samples were conducted. Hence, ethics approval was not required.

\section{Conflict of Interest Statement}

The authors have no conflicts of interest to declare.

\section{Funding Sources}

None.

\section{Author Contributions}

Guus C. Baan and Huub Maas conceived and designed the model; Guus C. Baan built the 3D model; Huub Maas reviewed and revised the $3 \mathrm{D}$ model; Huub Maas drafted the manuscript. Both authors reviewed and approved the final version of the manuscript.

\section{Data Availability}

All data used and models generated in this study are included in this article. Further enquiries can be directed to the corresponding author. 


\section{References}

Burkholder TJ, Nichols TR. Three-dimensional model of the feline hindlimb. J Morphol. 2004;261(1):118-29.

Charles JP, Cappellari O, Spence AJ, Hutchinson JR, Wells DJ. Musculoskeletal Geometry, Muscle Architecture and Functional Specialisations of the Mouse Hindlimb. PloS One. 2016;11(4):e0147669.

Corte GM, Humpenoder M, Pfutzner M, Merle R, Wiegard M, Hohlbaum K, et al. Anatomical Evaluation of Rat and Mouse Simulators for Laboratory Animal Science Courses. Animals (Basel). 2021;11(12):3432.

Crouch JE. Text-atlas of cat anatomy. Philadelphia: Lea \& Febiger; 1969.

Finni T, Bernabei M, Baan GC, Noort W, Tijs C, Maas H. Non-uniform displacement and strain between the soleus and gastrocnemius subtendons of rat Achilles tendon. Scand J Med Sci Sports. 2018;28(3):1009-17.
Greene EC. Anatomy of the rat. Transactions of the American Philosophical Society. New York: Hafner Pub.; 1935. p. 1-370.

Haberfehlner H, Maas H, Harlaar J, Becher JG, Buizer AI, Jaspers RT. Freehand three-dimensional ultrasound to assess semitendinosus muscle morphology. J Anat. 2016;229(4): 591-9.

Siebert T, Tomalka A, Stutzig N, Leichsenring K, Böl M. Changes in three-dimensional muscle structure of rabbit gastrocnemius, flexor digitorum longus, and tibialis anterior during growth. J Mech Behav Biomed Mater. 2017; 74:507-19.
Smilde HA, Vincent JA, Baan GC, Nardelli P, Lodder JC, Mansvelder HD, et al. Changes in muscle spindle firing in response to length changes of neighboring muscles. J Neurophysiol. 2016;115(6):3146-55.

Tijs C, van Dieën JH, Maas H. Limited mechanical effects of intermuscular myofascial connections within the intact rat anterior crural compartment. J Biomech. 2016;49(13):29539.

Young F, Rode C, Hunt A, Quinn R. Analyzing Moment Arm Profiles in a Full-Muscle Rat Hindlimb Model. Biomimetics (Basel). 2019; 4(1): 10 .

Zuurbier CJ, Everard AJ, Vanderwees P, Huijing PA. Length-Force Characteristics of the Aponeurosis in the Passive and Active Muscle Condition and in the Isolated Condition. J Biomech. 1994;27(4):445-53. 